Original Research Paper

\title{
Osmotic Tension, Plasticization and Viscoelastic response of amorphous Poly-Ether-Ether-Ketone (PEEK) equilibrated in humid environments
}

\author{
Aversa Raffaella and Apicella Antonio \\ Advanced Materials Lab, Department of Architecture and Industrial Design, Second University of Naples, Aversa, Italy
}

Article history

Received: 05-07-2016

Revised: 06-07-2016

Accepted: 14-07-2016

Corresponding Author: Apicella Antonio

Advanced Materials Lab, Department of Architecture and Industrial Design, Second University of Naples, Aversa, Italy

Email: antonio.apicella@unina2.it

\begin{abstract}
Dynamic-mechanical analysis (DMA), Differential scanning Calorimetry (DSC) and water moisture sorption-desorption kinetics and equilibria have been parallely investigated to monitoring the environmental and thermal sensitivity of amorphous thermoplastic Poly-Ether-EtherKetone (PEEK). Morphological modification occurring in PEEK during the calorimetric and thermo-mechanical characterizations and the hygrothermal stability are presented. An interpretation based on the free energy of mixing of a polymer chains with penetrant molecules and pseudo-equilibrium Flory-Huggins interaction parameter $\chi$ and related osmotic tension is proposed. The correlation between dynamic mechanical measurements and water saturation is discussed in terms of different levels of osmotic tension generated in the amorphous PEEK. From the analysis of the water diffusion coefficients in amorphous PEEK equilibrated at low and high activity environments, it has been found that even samples with very low water contents may undergo bulk matrix relaxation above $40^{\circ} \mathrm{C}$.
\end{abstract}

Keywords: PEEK, Water Sorption, Plasticization, Dynamic Mechanical Analysis, Viscoelasticity, Differential Scanning Calorimetry, FloryHuggins, Osmotic Tension

\section{Introduction}

The use of high performance engineering thermoplastics for advanced composites and application need a more deep understanding of the physical phenomena induced in the polymer during their lifespan operations in aggressive environments. In particular, it has been pointed out in previous publications (Apicella, 1990, Mensitieri et al., 1990) that several composite polymeric matrices may undergo mechanical and physical properties modifications due to the interaction with the external working environment. Hay and Kemmish (1988; Mensitieri et al., 1993) have reported solvent and moisture sorption sensitivity of Poly-Ether-Ether-Ketone (PEEK) in different temperature and penetrant characteristics, however, a physical interpretation and quantification of the possible properties degradation mechanism was not given.

The presence of the water molecules absorbed in the polymeric chain network has been found to significantly alters its thermal, mechanical and viscoelastic behaviors (Mikols et al., 1982; Pramoda and Liu, 2004; Velisaris and Seferis, 1986). The effect of the plasticizing penetrant molecules should be researched in the modification of the interaction between polymer-polymer chains that are substituted by the penetrant molecules polymer chains interactions.

Small molecules sorption could induce different levels of plasticization and swelling according to the amount of penetrant and to the intensity of the osmotic tensions generated by the penetrant-polymer pair (Sarti and Apicella 1980). The osmotic tension, $\pi_{o}$, differs from the more common osmotic pressure used to describe dilute polymer solutions in that it can be calculated through polymer partial molar quantities (Sarti and Apicella 1980, Reimhult et al., 2003). Osmotic tension in penetrant-polymer pairs, therefore, arises from the different penetrant molecules nature and concentration through the polymer and it can reach, depending on the distance from the glass transition, values of the order of tens of MPa (Sarti and Apicella, 1980). 
The physical phenomena that simultaneously could occur in an aggressive environment are dissolution, diffusion, swelling and relaxation, together with deformation, osmotic stress buildup and crystallization in the matrix. A wide variety of effects can be therefore exhibited depending on the mutual polymer-penetrant affinity, on the temperature and on the penetrant activity. Small molecules diffusion and solvent induced polymer relaxation and crystallization, in fact, are temperature-activated processes characterized by significantly different activation energies (Sarti and Apicella, 1980; Nicolais et al., 1984). Changes of the external temperature could modify the relative contributions of diffusion and relaxation leading to different sorption behavior and mechanical response. The present paper analyzes the plasticization and mechanical property decay by parallel interpretation of Dynamic Mechanical Analysis, Differential scanning Calorimetry and water moisture diffusion kinetics and equilibria.

\section{Materials, Apparatus and Procedures}

Materials

As received $25000 \mathrm{MW}$ amorphous Poly-EtherEther-Ketone APTIV PEEK-90 films have been used for sorption, calorimetric and dynamic mechanical characterizations. The density of the as received amorphous PEEK films has been measured at $25^{\circ} \mathrm{C}$ in a density gradient column and was $1.265 \mathrm{~g} / \mathrm{cm}^{3}$.

Double distilled water was used in the all sorption tests.

\section{Apparatus and Procedures}

\section{Thermal Analysis and Water Moisture sorptions}

\section{Differential Scanning Calorimetry:}

ADSC Mettler Differential Scanning Calorimeter has been used for the thermocalorimetric characterization. Temperature scans from 0 to $400^{\circ} \mathrm{C}$ were carried out at $5^{\circ} \mathrm{C} / \mathrm{min}$.

\section{Dynamic Thermo-Mechanical Characterization}

Dynamic-mechanical tests at different temperatures were carried out using a dynamic viscoelastic analyzer, Mettler DMA. Samples were tested in tensile load control mode at a frequency of $8 \mathrm{~Hz}$ by imposing static and dynamic loads of 0.5 and $0.05 \mathrm{MPa}$, respectively. Viscoelastic response data were measured in tensile load control mode during the isothermal pause of $20 \mathrm{~s}$ with temperature step of $10^{\circ} \mathrm{C}$ with an overall heating rate that was set both at 5 and $10^{\circ} \mathrm{C} / \mathrm{min}$. DMA tests performed on 2 $\mathrm{mm}$ amorphous PEEK samples dry and previously saturated in liquid water at $100^{\circ} \mathrm{C}$.

\section{Equilibrium Sorption and Desorption Tests}

A gravimetric quartz spring balance has been used to measure the equilibrium water uptakes in the PEEK films equilibrated at increasing water vapors relative pressures. In particular, all tests were made on $100 \mu \mathrm{m}$ amorphous PEEK films equilibrated at different vapor pressures in water vapor moist environments at 5, 20, 40 and $60^{\circ} \mathrm{C}$.

\section{Results and Discussion}

The thermal behavior of amorphous PEEK has been investigated both mechanically (DMA) and calorimetrically (DSC).

The DSC trace of a thermal scanrun at $5^{\circ} \mathrm{C} / \mathrm{min}$ on dry amorphous PEEK samples is shown in Fig. 1. The first thermal event occurring at the progressively heat-up is the glass transition that is observed in the range of temperature between $130-150^{\circ} \mathrm{C}$ (start and end of the glass transition, respectively). A clear exothermic peak is observed just above the glass transition.

Once in the rubbery state, in fact, when the PEEK macromolecular segments reach sufficient mobility to rearrange themselves in the more stable and thermodynamically favored spherulites crystalline state, a so called cold crystallization is abruptly activated at $160^{\circ} \mathrm{C}$ and it is evident as the sharp exothermic peak with its maximum at $170^{\circ} \mathrm{C}$. The cold crystallization process continues up to $250^{\circ} \mathrm{C}$ with crystallites lamellae thickening.

Finally a melting peak, which is characteristic of the semi-crystalline PEEK, is observed at $300-350^{\circ} \mathrm{C}$.

The viscoelastic thermo-mechanical behavior of amorphous PEEK samples has been preliminary determined in dynamic mechanical characterization from 0 to $250^{\circ} \mathrm{C}$. Tests were carried out every $10^{\circ} \mathrm{C}$ with an overall heating rate of $5 \% \mathrm{~min}$ using a Dynamic Mechanical Analyzer (DMA) operating in tension at a frequency of $8 \mathrm{~Hz}$ : The measured complex modulus and the loss factor (the ratio between the viscous and elastic components of the complex modulus) at the different testing temperatures are reported in Fig. 2.

The complex tensile modulus (upper curve in Fig. 2) continuously decreases from a value of about $2.0 \mathrm{GPa}$ at $0^{\circ} \mathrm{C}$ to $1.5 \mathrm{GPa}$ at $60^{\circ} \mathrm{C}, 1.3 \mathrm{GPa}$ at $100^{\circ} \mathrm{C}$ and $1.0 \mathrm{GPa}$ at $150^{\circ} \mathrm{C}$. The loss factor (lower curve in Fig. 2), which represents the ratio between the elastic and viscous mechanical response of the material at the different temperatures, is about 0.025 up to $150^{\circ} \mathrm{C}$ (namely, the sample is still glassy and it is essentially behaving elastically) and then it starts to increase to values that reach 0.3 at $250^{\circ} \mathrm{C}$.

The increase of the viscous component of the mechanical response above $150^{\circ} \mathrm{C}$ represents the incipient glass transition occurrence that has been 
described by Differential thermal analysis to occur in the $140-150^{\circ} \mathrm{C}$ range. Nevertheless, it has been observed that the complex modulus did not undergo the characteristic significant drop that isusually associated to the polymer softening at the glass transition.

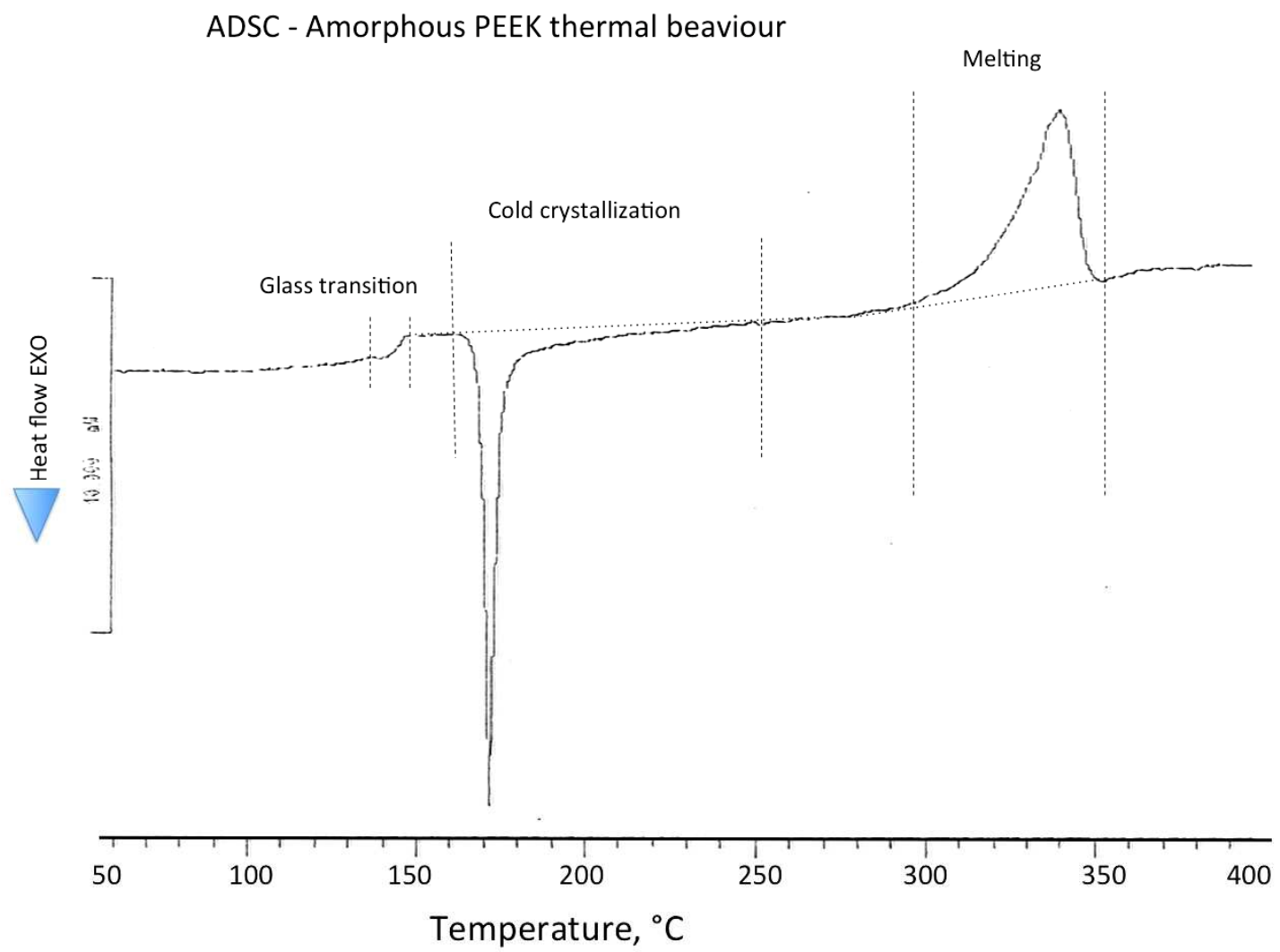

Fig. 1. Thermal behavior of dry amorphous PEEK sample from Differential Scanning Calorimetry run at $5^{\circ} \mathrm{C} / \mathrm{min}$

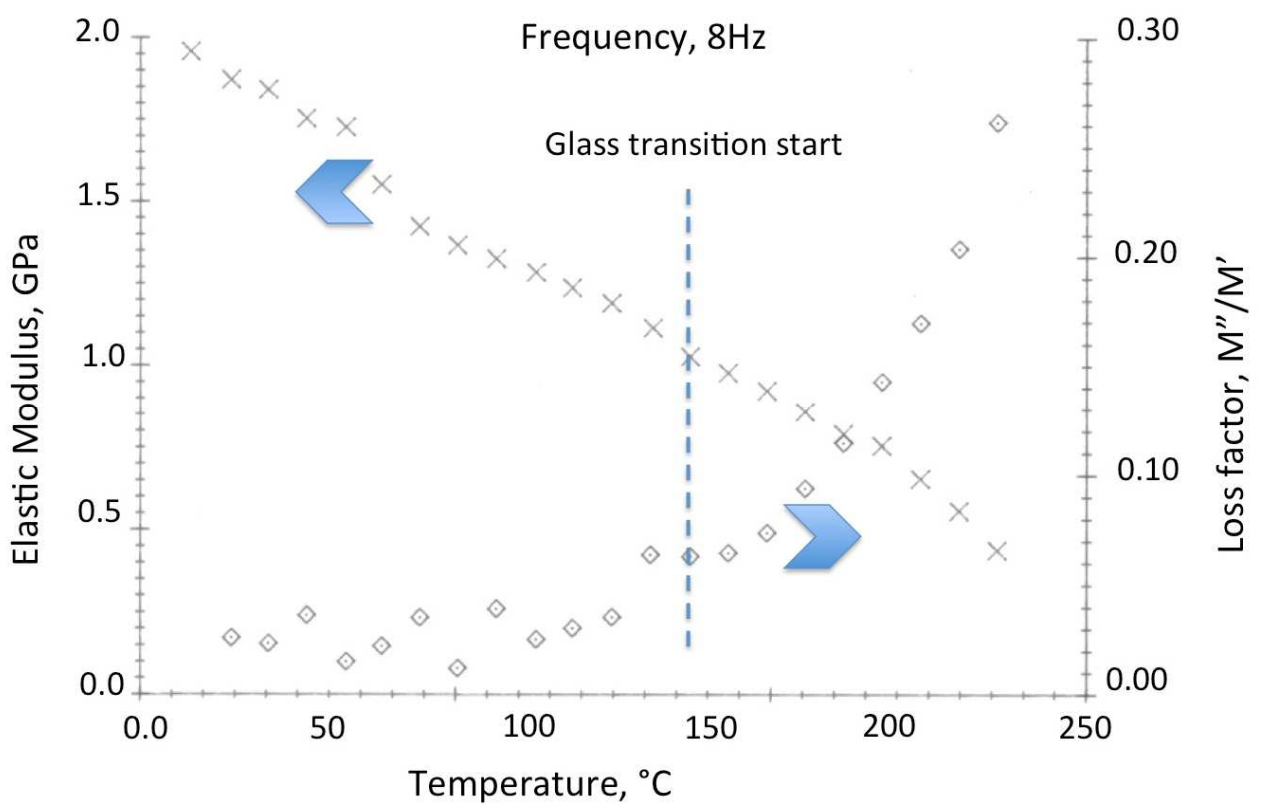

Fig. 2. Complex Modulus and Loss factor of amorphous PEEK in the Dynamic mechanical test 


\section{Plasticization of PEEK and Osmotic Tension in Water Moist Environments}

This phenomenon could be correlated to the cold crystallization thermal event observed from the DSC characterization. The progressively higher segmental mobility of the PEEK chains above its glass transition is hindered, as temperature is increased, by the formation of the crystalline phase that has been previously described by the DSC analysis to continue up to $250^{\circ} \mathrm{C}$. The crystalline lamellae thickening, that is a continuous process of melting and recrystallization of the neo-formed crystal phase, preserves a high viscous character that is evident in the continuous increase of the loss factor.

Conditioning and saturation in water moist environment has been observed in previous works to significantly alter the mechanical and viscoelastic response of several polymers used as matrices for advanced composites (Apicella, 1990; Mikols et al., 1982).

In order to investigate the moisture sensitivity of PEEK matrices in wet environments, the amorphous PEEK samples were saturated in water at $100^{\circ} \mathrm{C}$ and then characterized by Dynamic mechanical analysis in the range of temperatures from 50 to $250^{\circ} \mathrm{C}$. The overall heating rate of the thermo-mechanical analysis was set to $10^{\circ} \mathrm{C} / \mathrm{min}$ in order to minimize the effect of the water moisture desorption from the wet samples during the test (at least in the glassy state).

Figure 3 reports the influence of water saturation on the storage modulus (the elastic component of the complex modulus) and loss factor in PEEK samples in the dry and water-saturated states.
The complex shape of the curves in the region of the glass transition, as described earlier, depends on the cold crystallization and lamellae thickening processes occurring in the amorphous PEEK. Moreover, it can be inferred from Fig. 3 that moisture desorption from the wet samples become more significant as the test temperature is increased above the glass transition. The wet sample properties, in fact, tend progressively to those of the initially dry sample. Due to the sample thickness $(2 \mathrm{~mm})$ and the high heating rate, moisture desorption could be considered minimal when the sample is in its the glassy state and the effect of moisture on mechanical and viscoelastic properties correctly evaluated.

The PEEK water saturated sample is observed to significantly reduce its storage modulus in the glassy state compared tothe value in the dry state, namely, at $50^{\circ} \mathrm{C}$ the storage modulus drops from $1.45 \mathrm{GPa}$ in the dry state to $0.90 \mathrm{GPa}$ in the wet state. Moreover, the loss factor of the wet PEEK sample in the same temperature range is characterized by values higher than those of the dry samples $(0.20$ Vs 0.03 , respectively). The mechanical viscous response of the wet samples is about ten times higher than the viscous response of the dry samples in the dry state.

The glass transition start of dry and wet samples, which have been evaluated in Fig. 3 from the change in slope of the storage modulus curves at increasing temperatures, drops of about $15^{\circ} \mathrm{C}$ from 130 to $115^{\circ} \mathrm{C}$.

The strong plasticizing effect of moisture sorption could be attributed to the alteration of the interaction between polymer-polymer chains that are substituted by the water -polymer interactions.

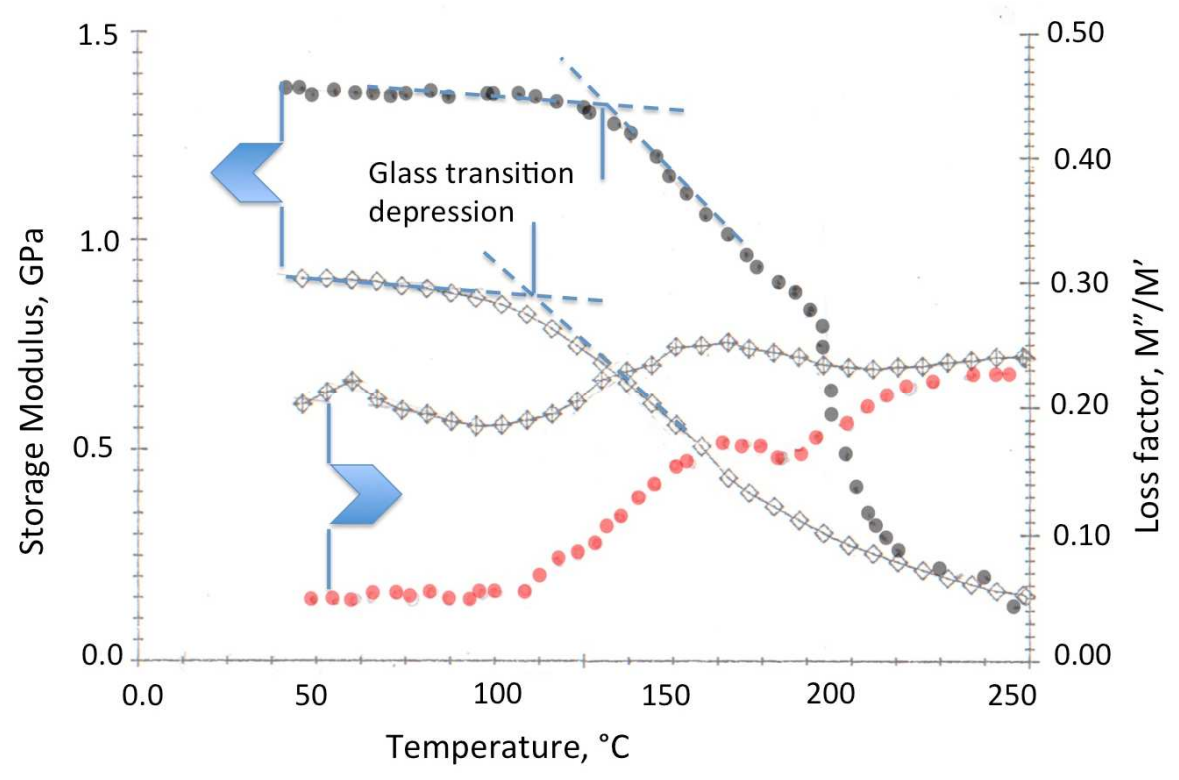

Fig. 3. Influence of water saturation on the Storage Moduli and Loss factors of amorphous PEEK in the Dynamic mechanical test 
The Flory-Huggins interaction parameter $\chi$ is a measure of the interactions of the polymeric chain segments with the penetrant molecules as well as the polymer-polymer interaction (Flory, 1953). Well below its glass transition, a glassy polymer can be considered in a pseudo-equilibrium state (D'Amore et al., 1990) and Flory-Huggins approach for polymer-solvent pair could be considered (Flory, 1953). The free energy of mixing of a polymer chains with penetrant molecules is described by Flory-Huggins theory considering the two contributions of the enthalpy of mixing, $\Delta H_{m i x}$ and the entropy of mixing, $\Delta S_{m i x}$. The entropy of mixing is calculated by the volume fractions of solvent and polymer, while the enthalpy of mixing is quantified by the dimensionless interaction parameter $\chi$.

The presence of the absorbed component in the polymer solid solution lowers the chemical potential of the guest molecule from its value in the pure condition. The evaluation of the chemical potential changes in differently water moisture saturated polymeric matrices has been used to calculate the osmotic tension rise due to the penetrant molecules sorption.

A theoretical expression for the reduction of the chemical potential of the penetrant molecules is derived from the free energy of mixing since the chemical potential of a penetrant molecule (solvent) in non-ideal solutions relative to that in its pure state relates to its activity trough the free energy of mixing (Flory, 1953):

$\mu_{1}-\mu_{1}^{\circ}=R T \ln a_{1}=R T\left[\ln \left(1-\varphi_{2}\right)+\left(1-\frac{1}{x}\right) \varphi_{2}+\chi_{1} \varphi_{2}^{2}\right] \quad 1$

where, $\mu_{1}^{\circ}$ is the pure penetrant molecule chemical potential, $\mu_{1}$ is penetrant molecule chemical potential in solid solution with polymer, $R$ is the gas constant and $T$ the absolute temperature. Moreover, $a_{1}$ is the activity of the dissolving molecules (which in our experiments was equal to the test relative water vapor pressure), $\varphi_{2}$ is the volume fraction of the polymer in the polymer-moisture equilibrium solution at the different water penetrant activities.

The interaction parameter $\chi_{1}$ accounts for the enthalpy changes occurring when polymer-polymer and penetrant-penetrant interactions are replaced by polymerpenetrant interactions.
Finally, $x$ is equal to $V_{2} / V_{1}$ with $V_{1}$ and $V_{2}$ the molar volumes of penetrant and polymer, respectively.

This term, which is very big for polymers/water systems since representing how many solvent molecules are needed to form the polymer macromolecule, lets to negligible $1 / \mathrm{x}$ values in Equation 1 that can be then written as:

$\ln a_{1}=\left[\ln \left(1-\varphi_{2}\right)+\varphi_{2}+\chi_{1} \varphi_{2}^{2}\right]$

This equation correlates water vapor activity in the sorption tests and the experimentally measured moisture uptakes.

Figure 4 reports the experimentally measured volume fractions of the moisture equilibrium uptakes reached at increasing water vapor activities in sorption tests run at $60^{\circ} \mathrm{C}$.

The full line in Fig. 4 represents the experimental data best fitting quadratic curve $\left(\varphi_{1}=0,0029 a^{2}\right.$ $+0,0086 a$ with a regression coefficient $\mathrm{R}^{2}=0,99412$ ) that will be used to evaluate from equation 3 the interactions parameters $\chi_{1}$ at different equilibrium water moisture contents.

Using the experimental equilibrium sorption best fitting curve, in fact, we may evaluate the interaction parameters, which should theoretically depend on the penetrant molecules concentration, at different moisture uptakes in the amorphous PEEK.

The calculated interaction parameters at different water activities for equilibrium sorption tests run at $60^{\circ} \mathrm{C}$ are reported in Table 1.

In particular, Table 1 lists the equilibrium water volume fractions $\varphi_{1}$, the Flory interaction parameter $\chi_{1}$ and the corresponding osmotic tensions $\pi_{0}$.

The Flory interaction parameter, which has been calculated from the best fitting isotherm equilibrium sorption curve, depends on the equilibrium concentration achieved at the different equilibration water activities: Their values range from 3.702 at the lower moisture concentration (with a water uptake of the $0.13 \%$ at an external water activity of 0.22 that corresponds to a volume fraction of 0.00164 ) to its minimum value of 3.558 for the maximum water activity of 1.0 (with a water uptake of $0.90 \%$ that corresponds to a volume fraction of 0.01150 ).

Table 1. Equilibrium water uptakes and corresponding Flory interaction parameters and pseudo-equilibrium osmotic tension

\begin{tabular}{lll}
\hline Water uptake, $\varphi_{1}$ & Flory-Huggins $\chi_{1}$ & Osmotic tension $\pi_{0}=\sigma, \mathrm{MPa}$ \\
\hline 0,01150 & 3.558 & $-22,35$ \\
0,01064 & 3.570 & $-20,14$ \\
0,00744 & 3.605 & $-14,45$ \\
0,00653 & 3.612 & $-13,47$ \\
0,00515 & 3.648 & $-9,09$ \\
0,00365 & 3.668 & $-6,94$ \\
0,00252 & 3.688 & $-5,11$ \\
0,00164 & 3.702 & $-3,94$ \\
\hline
\end{tabular}




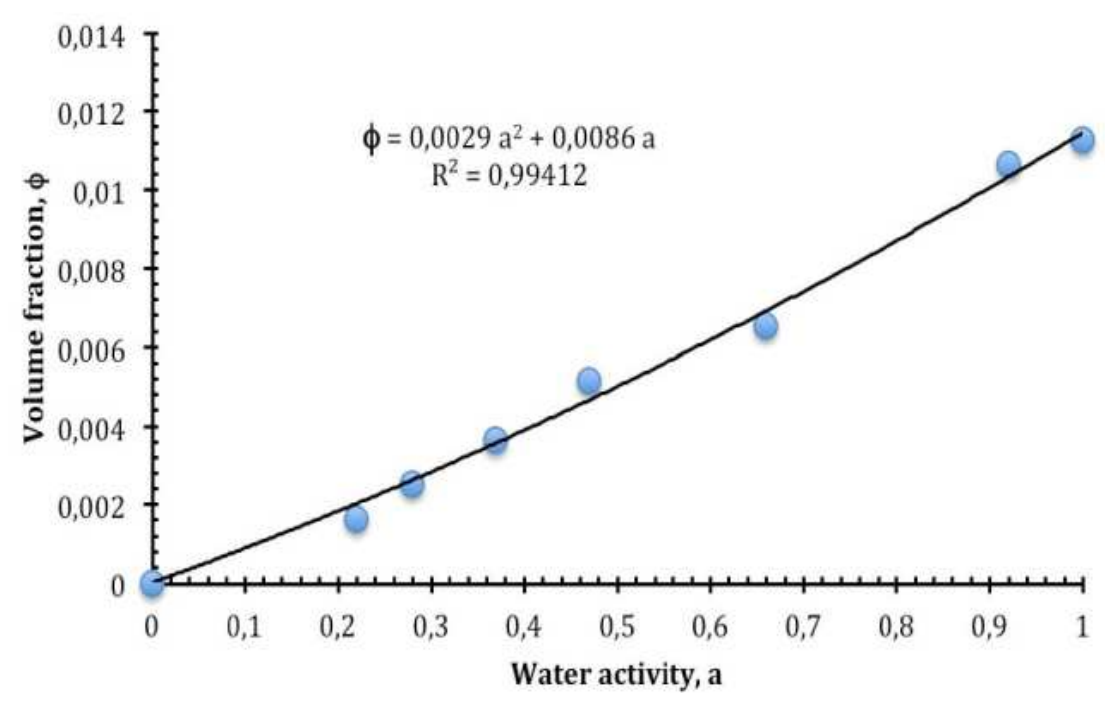

Fig. 4. Water sorption isotherm at $60^{\circ} \mathrm{C}$ in amorphous PEEK

By knowing the interaction parameters $\chi_{1}$ we may evaluate the osmotic expansion stresses generated by the presence of the water molecules sorption at different equilibrium moisture contents using this derivations:

$\sigma=\pi=-\frac{\left(\mu_{1}-\mu_{1}^{\circ}\right)}{V_{1}^{\circ}}$

where, $V_{1}^{0}$ is the water penetrant molecules molar volume, i.e., $18.07 \mathrm{~J}^{\mathrm{X}} \mathrm{mol}^{-1 \times} \mathrm{MPa}^{-1}$.

Rewriting equation 2 referred to equation 3 and to the watervolume fraction and accounting for the contribution of the $1 / x$, with $x$ the ratio $V_{1} / V_{2}$ between the molar volumes of the hosting polymer and the water penetrant, respectively 228,1 and 18,02 $\mathrm{ml} / \mathrm{mole}$ (Polymer database, web), we obtain:

$$
\pi=-\frac{\mathrm{RT}}{\mathrm{V}_{1}^{\mathrm{o}}}\left[\ln \left(1-\varphi_{1}\right)+\left(1-\frac{1}{x}\right) \varphi_{1}+\chi_{1} \varphi_{1}^{2}\right]
$$

The osmotic swelling pressures calculated from Equation 4 are reported in the last column of Table 1.

The calculated osmotic swelling pressures range from $3.94 \mathrm{MPa}$ at lower water contents (volume fraction 0,00164 when saturated at 0.22 water activity environment) to a maximum of $22,35 \mathrm{MPa}$ when saturated in liquid water (volume fraction 0,00164 when saturated at water activity $=1$ ). Such osmotic tensions could generate, especially at high temperatures, bulk polymer creep and swelling.

In Flory's theory, the isotropic stress generated by the sorbed molecules equates the total osmotic pressure to reach a static equilibrium state in the polymer. Namely, the penetrant swelling stress $(\sigma)$ in the polymer exerts a compressive pressure $(\pi)$ on the penetrant molecules; “... A close analogy exists between swelling equilibrium and osmotic equilibrium. The elastic reaction of the network structure may be interpreted as a pressure acting on the solution, or swollen gel...." (Flory, 1953).

These osmotic internal isotropic stresses reach values that are comparable with the amorphous PEEK bulk strength in the range of temperature $20-60^{\circ} \mathrm{C}$.

The corresponding values of the osmotic tensions reported in Table 1 should be considered as the maximum tension that could be generated in the case of a sharp front dividing two regions of the polymer where the diffusing penetrant molecules are absent and where the penetrant diffusing molecules reach a constant equilibrium volume fractions $\varphi_{1}$. Nevertheless, this situation occurs only for highly swollen polymer solvent pairs when limiting Case II sorption is observed (Holley et al., 1970; Sarti and Apicella, 1980).

At lower equilibrium penetrant molecules concentrations, when limiting Case II sorption mechanism is not observed, diffusing molecules concentration profiles are generated trough the polymer thickness.

According to ordinary Fickian diffusion, which is the other limiting sorption mechanism occurring when the diffusion coefficient does not depend on penetrant concentration, an analytically computed time dependent concentration profile though the polymer thickness could be derived and sorption kinetics could be described by known analytical expressions (Crank, 2004). The most common way to plot the sorption kinetics following Fick's law mechanism is to report the penetrant uptakes as a function of the square root of the sorption time normalized to the sample thickness. In such plots, the initial part of the sorption curve (or desorption curve if we start from saturated samples) is characterized by an initial linear trend which is proportional to the diffusion coefficient of the diffusing molecule in the host medium. 
Two sorption kinetics, that are representative of the sorption behaviors encountered in our experiment sat low and high activities, are reported in Fig. 5.

Sorption kinetic follows ordinary Fick's law at low external penetrant activity (lower curve in Fig. 5) while it moves out from the ordinary behavior when the water moisture content in the external environment is high. In particular, at high moisture contents (upper curve), the initial portion of the curve seems to follow ordinary Fickian diffusion up to the final part of the sorption whena deviation to higher uptakes is observed.

During the sorption transient, in fact, osmotic tensions of different intensities can be generated accordingly to the water concentration profile along the sample thickness, the higher is the equilibrium water uptake, the higher will be the osmotic tension generated in the penetrated polymer (see last column of Table 1).

Sorption and relaxation then occur simultaneously as the osmotic tensions are generated through the sample thickness by the penetrant molecules; the higher the equilibrium concentration of the penetrant, the higher the osmotic tension will be. In our case, as reported on the curve of Fig. 5, the osmotic tension generated in the sample equilibrated at the activity of $0.70(70 \% \mathrm{RH})$ is more than two times that generated at the activity 0.35 (35\% RH), 6,94 and 14,07 MPa, respectively. The Flory interaction parameters, which are also reported on the curves of Fig. 5, are 3.605 and 3.668 for the sorptions at high and low activities, respectively. The lower value at the high activity depends on higher affinity of the penetrant molecules in the plasticized polymer matrix.

In addition to the dependence on moisture content, osmotic tensions are also expected to generate different levels of swelling according to the viscoelastic response of the hosting polymer at the test temperature: Namely, the higher are the temperature and viscous character of the polymer mechanical response, the higher should be the swelling induced by the same osmotic loading conditions (the polymer creeps under the effect of the differential osmotic tension).

The viscoelastic response of amorphous PEEK in DMA experiments on dry and wet condition has been observed to depend both on temperature and equilibrium water content. It should be then expected that an increase of the viscous response of the hosting polymeric matrix (as it has been measured in our DMA test for plasticized PEEK) should result in a higher swelling of the penetrated regions and, hence in a progressive increase of the ability of the diffusing molecules to penetrate the polymer. This occurrence should affect the measured water diffusion coefficients leading to time and concentration dependency and to the anomalous sorption behavior observed in Fig. 5.

In order to evaluate the combined influence of water plasticization, osmotic tensions and polymer relaxation (swelling), diffusion coefficients in samples previously equilibrated at low water activities $(\mathrm{a}=0.35$ with water content of water weight $0.30 \%$ ) and high water activities ( $a=1.0$ with water weight content of $0.90 \%$ ) have been measured from desorption tests run at progressively higher temperatures.

It has been decided to use the desorption test to evaluate the diffusion coefficients in order to account either for the modifications induced in the matrix by the preliminary different levels of water saturation and for the influence of the test temperature on the relaxations occurring in the differently saturated samples (that were experienced different levels of osmotic tensions).

According to our model, due to the increased contribution of the viscous behavior at higher temperatures and moisture contents, higher swellings and, therefore, higher water diffusion coefficients should be expected in the amorphous PEEK both for higher temperatures and higher moisture contents.

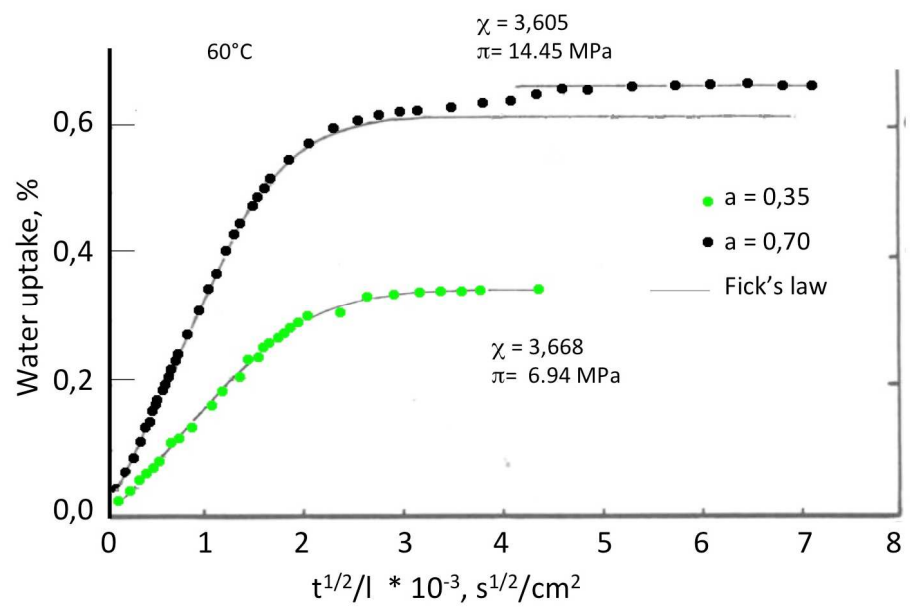

Fig. 5. Sorption behavior of water diffusion in amorphous PEEK at $60^{\circ} \mathrm{C}$ : Green dots, low activity sorption, black dots, high activity sorption 


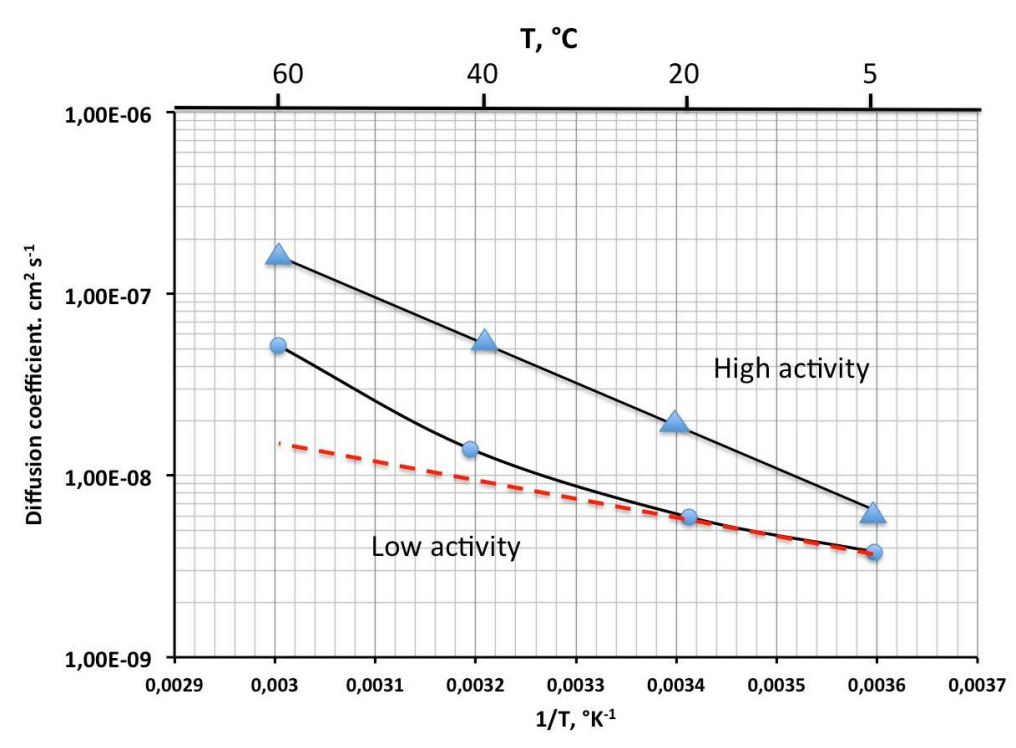

Fig. 6. Diffusion coefficients dependency on temperature for samples equilibrated in high and low activities environment

Figure 6 reports the diffusion coefficients measured at $5,20,40$ and $60^{\circ} \mathrm{C}$ when, according to our calorimetric and thermo-mechanical characterizations, the host matrix is still in its glassy state either for dry or water saturated samples.

Data are plotted in a semi-logarithmic diagram as a function of the inverse of the absolute temperature according to the temperature dependence of thermally activated process:

$$
D=D_{0} \exp \left(-E_{a} / R T\right)
$$

where, $D_{0}$ is a constant pre-exponential factor and $\mathrm{E}_{\mathrm{a}}$ is the process activation energy.

This relationship holds only for constant diffusion coefficients that are independent by the penetrant concentration.

The diffusion coefficients of the samples saturated at high water activity show a linear dependency on the inverse of the absolute temperature (as it should be expected for an activated process).

Conversely, the diffusion coefficients measured for the samples equilibrated at the low activity do not follow this linear behavior.

As it can be inferred from Fig. 6, the diffusion coefficients evaluated for the samples equilibrated at low activity and, hence, characterized by a lower water content, strongly deviate from the expected linear behavior at 40 and $60^{\circ} \mathrm{C}$ (red dotted line).

At $60^{\circ} \mathrm{C}$ the measured value is 4 times higher than the expected one; this occurrence is the evidence of the significant swelling of the hosting matrix that is induced by the osmotic tension acting in the plasticized and more viscous glassy matrix (with a higher complex modulus viscous component).
Conversely, in the samples saturated at high water contents (at higher water external activities) these osmotic tensions were sufficiently high to induce matrix relaxation and swelling even at lower test temperatures.

\section{Conclusion}

In the last decade a large number of new applications of PEEK engineering polymers have been developed. These include high performance aircraft and automotive devises as well as appliance components.

This eclectic polymer may then experience a wide number of temperature and aggressive environment operational conditions. Some of them or combination of them could seriously modify its physical and mechanical characteristics.

The understanding of the mechanisms of mechanical and thermal resistance of PEEK in humid environment under variable temperature and sorbent loading conditions it is an important issue to improve the reliability of PEEK structural and functional components.

A model has been proposed to evaluate the influence of water sorption on PEEK environmental aging in aggressive environments where temperature and presence of sorbent species could significantly alter the PEEK component reliability.

\section{Ethics}

No ethical issues arise from this paper.

\section{Author's Contributions}

Prof. Aversa Raffaella, run all characterization tests and data theoretical interpretation. Prof. Apicella investigated some of the base theoretical aspects. 


\section{References}

Apicella, A., 1990. Environmental Resistance of HighPerformance Polymeric Matrices and Composites. In International Encyclopedia of Composites, Lee, S. (Ed.), VCH, Publisher, ISBN-10: 0-89573-732-9, pp: 46-67.

Crank, J., 2004. The Mathematics of Diffusion. 2nd Edn., Oxford Science Publications, Oxford, England, ISBN-13: 978-0198534112.

D'Amore, A., F. Cocchini, A. Pompo, A. Apicella and L. Nicolais, 1990. Effect of physical aging on longterm properties of poly-ether-ketone (PEEK) and PEEK-based composites. J. Applied Polymer Sci., 39: 1163-1174. DOI: 10.1002/app.1990.070390511

Flory, P.J., 1953. Principles of Polymer Chemistry. 1st Edn., Cornell University Press, ISBN-10: 0801401348, pp: 672.

Hay, J.N. and D.J. Kemmish, 1988. Environmental stress crack resistance of and absorption of low-molecularweight penetrants by poly(aryl ether ether ketone). Polymer, 29: 613-618. DOI: $10.1016 / 0032-3861(88) 90074-2$

Holley, R.H., H.B. Hopfenberg and V. Stannett, 1970. Anomalous transport of hydrocarbons in polystyrene. Polymer Eng. Sci., 10: 376-382. DOI: $10.1002 /$ pen.760100612

Mensitieri, G., A. Apicella, M.A. Del Nobile and L. Nicolais, 1993. Extreme environmental resistance of PEEK matrix. J. Reinforced Plast. Comp., 12: 1138-1149. DOI: 10.1177/073168449301201101

Mensitieri, G., A. Apicella, M.A. Del Nobile, L. Nicolais and F. Garbassi, 1990. Solvent induced crystallization in poly(aryl-ether-ether-ketone). J. Mater. Sci., 25: 2963-2970.

DOI: $10.1007 / \mathrm{BF} 00584912$
Mikols, W.J., J.C. Seferis, A. Apicella and L. Nicolais, 1982. Evaluation of structural changes in epoxy systems by moisture sorption-desorption and dynamic mechanical studies. Polymer Comp., 3: 118-124. DOI: $10.1002 /$ pc. 750030304

Nicolais, L., A. Apicella and C. Denotaristefano, 1984. Time-temperature superposition of n-hexanesorption in polystyrene. J. Membrane Sci., 18: 187-196. DOI: $10.1016 / \mathrm{S} 0376-7388(00) 85033-4$

Pramoda, K.P. and T. Liu, 2004. Effect of moisture on the dynamicmechanicalrelaxation of polyamide6/claynanocomposites. Polym Sci. Part B, 42: 1823-1830. DOI: 10.1002/polb.20061

Reimhult, E., F. Höök and B. KasemoIntact, 2003. Intact vesicle adsorption and supported biomembrane formation from vesicles in solution: Influence of surface chemistry, vesicle size, temperature and osmotic pressure. Langmuir, 19: 1681-1691. DOI: $10.1021 / \mathrm{la} 0263920$

Sarti, G.C. and A. Apicella, 1980. Nonequilibrium glassy properties and their relevance in case II transport kinetics. Polymer, 21: 1031-1036. DOI: 10.1016/0032-3861(80)90033-6

Velisaris, C.N. and J.C. Seferis, 1986. Crystallization kinetics of polyetheretherketone (peek) matrices. Polymer Eng. Sci., 26: 1574-1581. DOI: $10.1002 /$ pen. 760262208 